\title{
The prognostic value of optic nerve sheath diameter in patients with subarachnoid hemorrhage
}

\author{
Sangkil Lee ${ }^{1}$, Yong Oh Kim², Ji Sun Baek ${ }^{3}$ and Jeong-Am Ryu $2,4^{*}$
}

\begin{abstract}
Background: We evaluated the role of optic nerve sheath diameter (ONSD) using brain computed tomography $(C T)$ in predicting neurological outcomes of patients with subarachnoid hemorrhage (SAH).

Methods: This was a retrospective, multicenter, observational study of adult patients with SAH admitted between January 2012 and June 2017. Initial brain CT was performed within $12 \mathrm{~h}$ from onset of SAH, and follow-up brain CT was performed within $24 \mathrm{~h}$ from treatment of a ruptured aneurysm. Primary outcome was neurological status at 6-month follow-up assessed with the Glasgow Outcome Scale (GOS, 1 to 5).

Results: Among 223 SAH patients, 202 (90.6\%) survived until discharge. Of these survivors, 186 (83.4\%) manifested favorable neurological outcomes (GOS of 3, 4, or 5). In this study, the ONSDs in the group of patients with poor neurological outcome were significantly greater than those in the favorable neurological outcome group (all $p<0.01$ ). Intracranial pressure (ICP) was monitored in 21 (9.4\%) patients during the follow-up CT. A linear correlation existed between the average ONSD and ICP in simple correlation analysis $(r=0.525, p=0.036)$. Analysis of the receiver operating characteristic curve for prediction of poor neurological outcome showed that ONSD had considerable predictive value (C-statistics, 0.735 to 0.812 ). In addition, the performance of a composite of Hunt and Hess grade and ONSD was increasingly associated with poor neurological outcomes than the use of each marker alone.

Conclusions: ONSD measured with CT may be used in combination with clinical grading scales to improve prognostic accuracy in SAH patients.
\end{abstract}

Keywords: Optic nerve sheath diameter, Brain computed tomography, Subarachnoid hemorrhage

\section{Key messages}

- In this study, patients with poor-grade SAH showed considerable survival (79.5\%) and favorable neurological prognoses (61.4\%).

- Concurrent measurement of ONSDs using brain CT and ICP was moderately correlated.
- The ONSDs of patients with poor neurological outcome were significantly greater than those with a favorable neurological outcome.

- A composite parameter of H-H grade and ONSD measured with CT may increase the accuracy of prognosis in patients diagnosed with $\mathrm{SAH}(\mathrm{H}-\mathrm{H}$ grade $>3$ and follow-up $\mathrm{ONSD}_{\text {index }}>6.22$ ).

\section{Background}

Subarachnoid hemorrhage (SAH) is a complex neurovascular syndrome and a devastating condition associated with high mortality and morbidity rates for those who survive the initial hemorrhage [1-3]. In SAH patients, the level of consciousness at admission is the most critical early predictor of clinical outcome [1, 4]. Patients with poor-grade SAH (grades 4 to 5) based on Hunt and

\footnotetext{
* Correspondence: lamyud.ryu@samsung.com

${ }^{2}$ Department of Critical Care Medicine, Samsung Medical Center,

Sungkyunkwan University School of Medicine, 81 Irwon-ro, Gangnam-gu,

Seoul 06351, Republic of Korea

${ }^{4}$ Department of Neurosurgery, Samsung Medical Center, Sungkyunkwan

University School of Medicine, 81 Irwon-ro, Gangnam-gu, Seoul 06351,

Republic of Korea

Full list of author information is available at the end of the article
} 
Hess [5] or World Federation of Neurosurgical Societies (WFNS) grading scales [6] show high mortality and poor neurological outcomes [7]. However, recent reports suggest that patients with poor-grade SAH treated early and aggressively with coil embolization and neurointensive care achieved favorable neurological outcomes [3, 7]. Therefore, new predictors other than the initial level of consciousness are needed to accurately evaluate the prognosis of these patients.

Poor neurological outcomes of SAH patients are usually secondary to early brain injury, rebleeding, or delayed cerebral ischemia $[1,8,9]$. Early brain injury is associated with intracranial hypertension $[1,9,10]$. Therefore, early monitoring of intracranial hypertension may allow prediction of neurological outcomes in these patients. Optic nerve sheath diameter (ONSD) has been proposed as an alternative measure for the detection of intracranial hypertension $[11,12]$. The ONSD may be associated with neurological outcomes of SAH patients. However, it has not been reported whether ONSD may facilitate systemic evaluation of neurological outcomes of patients with SAH. Therefore, the objective of this study is to investigate if ONSD with some modifications could be used to predict neurological outcomes of patients with SAH.

\section{Methods}

\section{Study population and design}

This was a retrospective, multicenter, observational study of adult patients with SAH admitted to the neurosurgical intensive care unit at ChungBuk National University Hospital (CBNUH) and Samsung Medical Center (SMC) between January 2012 and June 2017. The study was approved by the institutional review boards of CBNUH (CBNUH 2018-08-014-001) and SMC (SMC 2018-07-154). The requirement for informed consent was waived due to the retrospective study design. We included patients with (1) SAH admitted to the neurosurgical intensive care unit during the study period, (2) brain computed tomography (CT) within $12 \mathrm{~h}$ from the onset of SAH, (3) follow-up brain CT within $24 \mathrm{~h}$ of treatment for ruptured aneurysm, and (4) follow-up brain CT within $48 \mathrm{~h}$ from the onset of SAH if they were not treated for aneurysm. Of these patients, we excluded patients (1) under age 18; (2) with malignancy and whose expected life span was less than 1 year; (3) with insufficient medical records; (4) with a history of head trauma, neurosurgery, cardiac arrest, or chronic neurological abnormality on admission; (5) transferred from other hospitals after more than $24 \mathrm{~h}$ of SAH onset; or (6) with orbital anomaly, orbital mass lesions, and ocular or retro-orbital injury. We analyzed a total of 223 patients diagnosed with SAH in this study (Fig. 1).

\section{Measurements and outcomes}

In this study, we defined poor-grade $\mathrm{SAH}$ as grades 4 to 5 according to Hunt and Hess $(\mathrm{H}-\mathrm{H})[1,4,5]$. The primary outcome was neurological status 6 months later assessed with a Glasgow Outcome Scale (GOS, 1 to 5) [12]. Patients with GOS scores of 3, 4, and 5 indicated

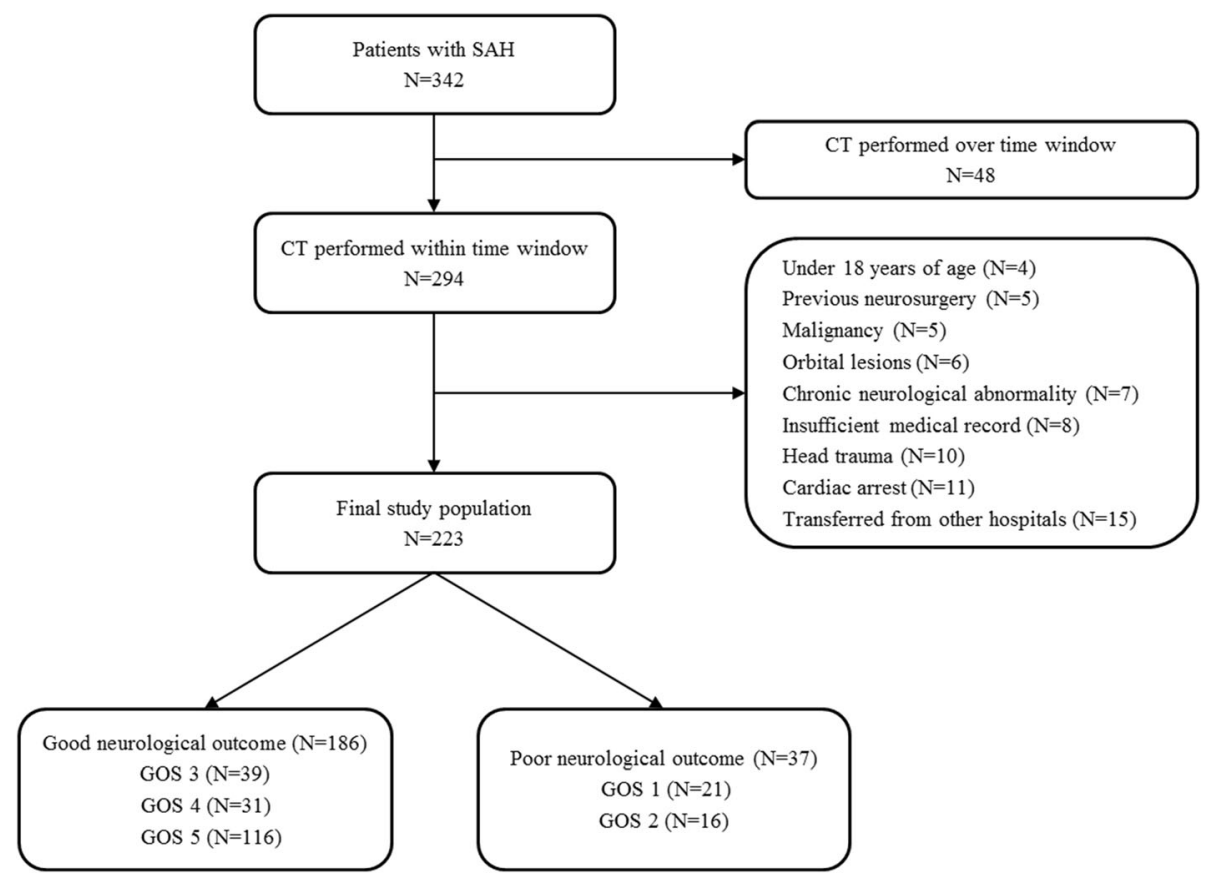

Fig. 1 Study flow chart. SAH subarachnoid hemorrhage, CT computed tomography, GOS Glasgow Outcome Scale 
favorable neurological outcomes whereas GOS scores of 1 and 2 indicated poor neurological outcomes. We thoroughly reviewed medical records, and two independent neurologists measured the patients' GOS scores. Initial brain CT angiography and follow-up CT were performed within a specified time window. All the CT studies were performed using 64-channel scanners (Brilliance 64, Philips Medical Systems, Best, the Netherlands, at CBNUH and Light Speed VCT, GE Healthcare, Milwaukee, WI, USA, at SMC) with a 5-mm-slice width. Brain CT images were reviewed by two independent neurologists (SL and JAR). Investigators who were blinded to clinical information evaluated each of the patients' CT scans using commercial image-viewing software (Picture Archiving and Communication System; Maroview 5.3 Infinitt Co., Seoul, Republic of Korea, at CBNUH and Centricity RA1000 PACS Viewer, GE Healthcare at SMC). The ONSD and eyeball transverse diameter (ETD) were measured using the same initial CT and subsequent scans. The ONSD was measured at a distance of $3 \mathrm{~mm}$ behind the eyeball, immediately below the sclera in a perpendicular vector with reference to the linear axis of the nerve (Fig. 2a) [11-13]. The images were changed to the "chest/abdomen" window (window width 300 and window level 10) and magnified fourfold on the particular image slice that demonstrated the largest diameter of the optic nerve sheath [12]. The ONSD was measured from one side of the optic nerve sheath to the other as a section through the center of the optic nerve [13]. The transverse diameter of the eyeball was chosen because the ONSD is usually measured in the transverse plane [14]. ETD was defined as the maximal transverse diameter of the eyeball measured from one side of the retina to the other (in-to-in, Fig. 2b) [14, 15]. The $\mathrm{ONSD}_{\text {average }}$ and the ETD $_{\text {average }}$ measured for the patient's left and right eyes were averaged to yield a mean value. The $\mathrm{ONSD}_{\text {index }}$ was defined as median ETD $(22.7 \mathrm{~mm})$ multiplied by the average value of bilateral ONSDs divided by the average value of bilateral ETDs $\left(22.7 \times \mathrm{ONSD}_{\text {average }} / \mathrm{ETD}_{\text {average }}\right)$. The intracranial pressure (ICP) at the time of follow-up CT was designated as the immediate ICP after the CT scan.

\section{Statistical analyses}

Clinical and laboratory data were collected by a trained study coordinator using a standardized case report form. All data are presented as means \pm standard deviations (SD) for continuous variables and numbers (percentages) for categorical variables. We compared data using the Student's $t$-test for continuous variables and chi-square test or Fisher's exact test for categorical variables. A scatter plot was drawn to ascertain the relationship between simultaneously measured ONSD and ICP. We calculated Pearson's correlation coefficient $(r)$ to evaluate the correlation between ONSD and ICP. We assessed the predictive performance of ONSDs and ONSD indices using the areas under the curve (AUCs) of the receiver operating characteristic (ROC) curves for sensitivity vs. 1-specificity. We compared AUCs using the nonparametric approach published by DeLong et al. [16] for two correlated AUCs. We obtained optimal cutoffs for each ONSD and their modifications to predict poor neurological outcomes by ROC curve and Youden index $[17,18]$. We used the bidirectional elimination technique in the stepwise selection method to select clinically and statistically meaningful predictor variables. The adequacy of the prediction model was also determined using the Osius and Rojek test of goodness-of-fit [19]. All tests were two-sided, and $p<0.05$ was statistically significant. We analyzed the data using IBM SPSS version 20 (IBM, Armonk, NY, USA).

\section{Results}

Baseline characteristics and clinical outcomes

The mean age of patients was $58.7 \pm 13.0$ years, and 84 (37.7\%) patients were males. Hypertension (39.5\%) and smoking (26.9\%) were the most common comorbidities
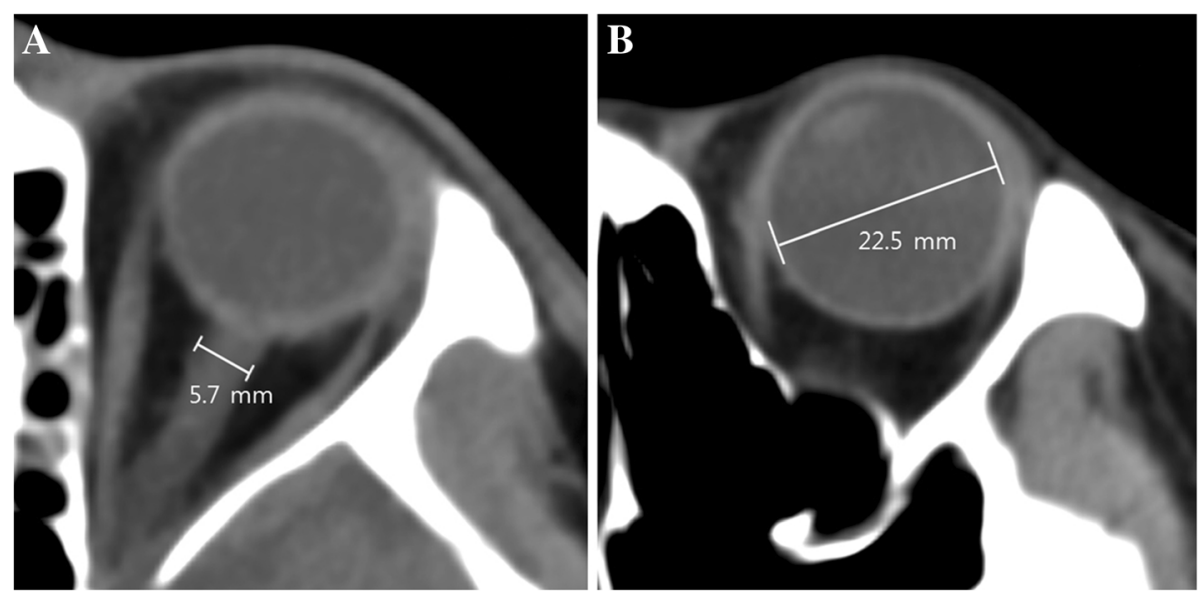

Fig. 2 Measurement of optic nerve sheath diameter (a) and eyeball transverse diameter (b) on the brain computed tomography scan 
among the SAH patients. Eighty-three (37.2\%) patients were $\mathrm{H}-\mathrm{H}$ grades 4 or 5 , and $96(43.0 \%)$ were WFNS grades 4 or 5 . WFNS grade was higher in patients included under the poor neurological outcome group compared with the favorable neurological outcome group ( $4.4 \pm 1.1$ vs. $2.4 \pm 1.5, p<0.001)$. Fisher grade and modified Fisher grade were also higher in the poor neurological outcome group compared with favorable neurological outcome group $(3.9 \pm 0.3$ vs. $3.2 \pm 0.7, p<0.001$ and $3.8 \pm 0.4$ vs. $2.9 \pm 1.0, p<0.001$, respectively). Baseline characteristics of SAH patients are presented in Table 1. Anterior communicating artery $(29.6 \%)$ and middle cerebral artery $(25.1 \%)$ were the most common locations of a ruptured aneurysm. However, no aneurysm or ruptured aneurysm was detected in 14 patients (6.3\%). Ruptured aneurysm was treated within $72 \mathrm{~h}$ in most patients (88.8\%). Endovascular coiling of the ruptured aneurysm was performed in $124(55.6 \%)$ patients, and surgical clipping was performed in 75 (33.6\%) patients; delayed cerebral ischemia was observed in 46 (20.6\%) patients. Treatment characteristics of SAH patients are presented in Table 2. Two hundred two patients $(90.6 \%)$ survived to discharge. Of these 202 survivors, 186 (83.4\%) had favorable neurological outcomes (GOS of 3, 4, or 5, Fig. 1). Among the 83 (37.2\%) patients with poor-grade SAH, 66 survived to discharge (79.5\%), and $51(61.4 \%)$ had favorable neurological outcomes.

\section{Optic nerve sheath diameters and their modifications}

In this study, initial and follow-up ONSDs in the poor neurological outcome group were significantly greater than in the favorable outcome group (Table 3). However, initial ETD and follow-up ETD did not differ significantly between the favorable and poor neurological outcome groups $(P=0.504$ and $P=0.295$, respectively). In addition, changes in ONSD $\left(\triangle \mathrm{ONSD}_{\text {average }}\right)$ between initial and follow-up CT were not associated with prognosis. Although invasive ICP monitoring was performed in $80(35.9 \%)$ patients during hospitalization, the ICP was measured in only $21(9.4 \%)$ patients at the time of follow-up CT. Based on simple correlation analysis, the ICP showed a linear correlation with follow-up ONS$\mathrm{D}_{\text {average }}$ and follow-up $\mathrm{ONSD}_{\text {index }}(r=0.525, P=0.036$ and $r=0.500, P=0.048$, respectively) (Fig. 3). In ROC curve analysis for prediction of poor neurological outcomes (Fig. 4a), the AUCs of ONSD ${ }_{\text {index }}$ were greater than those of $\mathrm{ONSD}_{\text {average }}$ during the initial $\mathrm{CT}$ and follow-up CT. However, no significant differences existed between the predictive performance of $\mathrm{ONSD}_{\mathrm{in}}$ dex and $\mathrm{ONSD}_{\text {average }}$ during the initial CT and follow-up CT $(P=0.764, P=0.086$, respectively). The performance of $\mathrm{ONSD}_{\text {index }}$ in follow-up CT was significantly better than that of $\mathrm{ONSD}_{\text {index }}$ and $\mathrm{ONSD}_{\text {average }}$ in initial CT $(P=0.033, P=0.022$, respectively). The $C$-statistic of follow-up $\mathrm{ONSD}_{\text {index }}$ was 0.812 ( $95 \%$ confidence interval (CI) 0.754 to 0.861 ). A cutoff $>6.22$ had a sensitivity of $70.3 \%$ (95\% CI 53.0 to $84.1 \%$ ) and a specificity of $80.7 \%$ (95\% CI 74.2 to $86.1 \%$ ). The $\mathrm{C}$-statistic of $\mathrm{H}-\mathrm{H}$ grade was 0.853 ( $95 \%$ CI 0.799 to 0.897 ). A cutoff $>3$ had a sensitivity of $86.5 \%$ ( $95 \%$ CI 71.2 to $95.5 \%$ ) and a specificity of $72.6 \%$ (95\% CI 65.6 to $78.9 \%$ ). Although no significant differences existed between the AUCs of $\mathrm{H}-\mathrm{H}$ grade, the follow-up $\mathrm{ONSD}_{\text {average, }}$ and the follow-up ONSD $_{\text {index }}$, the composite of $\mathrm{H}-\mathrm{H}$ grade and the follow-up $\mathrm{ONSD}_{\text {index }}$ [AUC 0.896, Brier score 0.078, goodness-of-fit (Osius-Rojek) $Z=-0.5052, \quad p=0.613$ ] showed the highest ability to correctly predict poor neurological outcomes rather than either marker alone (all $p<0.006$, Fig. 4b). Based on our definitions of poor markers as $\mathrm{H}-\mathrm{H}$ grade $>3$ and follow-up $\mathrm{ONSD}_{\text {index }}>$ 6.22, 107 (48.0\%) patients had one or more poor markers (sensitivity $91.9 \%$ and specificity $60.8 \%$ ). Of these patients, $39(17.5 \%)$ carried two poor markers $(\mathrm{H}-\mathrm{H}$ grade $>3$ [poor-grade $\mathrm{SAH}$ ] and follow-up $\mathrm{ONSD}_{\text {index }}>6.22$, sensitivity $64.9 \%$, and specificity $91.9 \%$ ).

\section{Discussion}

In this study, we evaluated whether ONSDs and their modifications could be used to predict neurological outcomes of patients with SAH. The major study findings were as follows: (1) Patients with poor-grade SAH manifested considerable survival rate $(79.5 \%)$ and favorable neurological prognoses (61.4\%); (2) simultaneous measurements of ONSDs with brain CT and ICP were moderately correlated; (3) during initial and follow-up CT, the ONSDs of patients in the poor neurological outcome group were significantly greater than in those of the favorable neurological outcome group, and these markers might predict neurological outcomes of SAH patients. In addition, follow-up ONSDs predicted prognosis better than the initial ONSDs; (4) the combined $\mathrm{H}-\mathrm{H}$ grade and ONSD measured with CT was strongly associated with poor neurological outcomes than the use of either marker alone $(\mathrm{H}-\mathrm{H}$ grade $>3$ [poor-grade $\mathrm{SAH}$ ] and follow-up $\mathrm{ONSD}_{\text {index }}>6.22$ ).

Although it is difficult to predict the outcomes following SAH, the level of consciousness at admission is associated with patient prognosis $[1,3,7]$. The widely accepted predictors include $\mathrm{H}-\mathrm{H}$ grade [5], WFNS grade based on the level of consciousness assessed using the Glasgow coma scale $[6,7]$, and more recently, VASOGRADE-Red [20]. In particular, patients with poor-grade SAH historically showed high mortality and poor neurological outcomes [7]. However, in recent years, a considerable number of patients with poor-grade SAH treated aggressively with early coil embolization and supportive neurointensive care achieved favorable neurological outcomes and survival 
Table 1 Baseline characteristics

\begin{tabular}{|c|c|c|c|}
\hline & Favorable neurological outcome $(n=186)$ & Poor neurological outcome $(n=37)$ & $p$ value \\
\hline Age (year)—mean $\pm S D$ & $57.7 \pm 12.1$ & $63.8 \pm 16.0$ & 0.034 \\
\hline Gender, male—no. of patients (\%) & $69(37.1 \%)$ & $15(40.5 \%)$ & 0.834 \\
\hline $\mathrm{BMI}\left(\mathrm{kg} / \mathrm{m}^{2}\right)$-mean $\pm \mathrm{SD}$ & $23.7 \pm 3.8$ & $22.8 \pm 3.5$ & 0.219 \\
\hline \multicolumn{4}{|l|}{ Comorbidities—no. of patients (\%) } \\
\hline Hypertension & $73(39.2)$ & $15(40.5)$ & 0.999 \\
\hline Current smoker & $51(27.4)$ & $9(24.3)$ & 0.853 \\
\hline Diabetes mellitus & $10(5.4)$ & $7(18.9)$ & 0.013 \\
\hline Dyslipidemia & $10(5.4)$ & $4(10.8)$ & 0.382 \\
\hline Previous TIA or stroke & $6(3.2)$ & $5(13.5)$ & 0.026 \\
\hline Ischemic heart disease & $6(3.2)$ & $4(10.8)$ & 0.109 \\
\hline Malignancy & $6(3.2)$ & $2(5.4)$ & 0.867 \\
\hline Chronic kidney disease & $4(2.2)$ & $3(8.1)$ & 0.167 \\
\hline The interval from symptom onset to initial $\mathrm{CT}$ & $4.5 \pm 5.9$ & $2.7 \pm 3.4$ & 0.015 \\
\hline The interval from symptom onset to follow-up CT & $26.5 \pm 13.4$ & $24.5 \pm 13.4$ & 0.385 \\
\hline The interval from initial CT to follow-up CT & $22.0 \pm 12.1$ & $21.7 \pm 13.7$ & 0.876 \\
\hline Hunt and Hess classification—-no. of patients (\%) & & & $<0.001$ \\
\hline 2 & $98(52.7)$ & $2(5.4)$ & \\
\hline 3 & $37(19.9)$ & $3(8.1)$ & \\
\hline 4 & $29(15.6)$ & $7(18.9)$ & \\
\hline 5 & $12.1 \pm 3.9$ & $6.4 \pm 3.6$ & \\
\hline Modified Fisher classification—no. of patients (\%) & & & $<0.001$ \\
\hline 1 & $29(15.6)$ & $0(0)$ & \\
\hline 2 & $10(5.4)$ & $0(0)$ & \\
\hline 3 & $101(54.3)$ & $9(24.3)$ & \\
\hline 4 & $46(24.7)$ & $28(75.7)$ & \\
\hline Pupil reactivity—no. of patients (\%) & & & $<0.001$ \\
\hline Both intact pupil reflex & $166(89.2)$ & $15(40.5)$ & \\
\hline One unreactive pupil & $4(2.2)$ & $3(8.1)$ & \\
\hline Both unreactive pupil & $16(8.6)$ & $19(51.4)$ & \\
\hline Aneurysm location—no. of patients (\%) & & & 0.015 \\
\hline Anterior communicating artery & $51(24.4)$ & $11(29.7)$ & \\
\hline Anterior cerebral artery and distal & $13(7.0)$ & $2(5.4)$ & \\
\hline Middle cerebral artery and distal & $51(27.4)$ & $5(13.5)$ & \\
\hline Internal carotid artery & $11(5.9)$ & $9(24.3)$ & \\
\hline Posterior communicating artery & $24(12.9)$ & $3(8.1)$ & \\
\hline Posterior circulation & $22(11.8)$ & $2(5.4)$ & \\
\hline No aneurysm & $10(5.4)$ & $4(10.8)$ & \\
\hline Unknown & $4(2.2)$ & $1(2.7)$ & \\
\hline Hydrocephalus_-no. of patients (\%) & $85(45.7)$ & $26(70.3)$ & 0.011 \\
\hline Intraventricular hemorrhage_-no. of patients (\%) & $54(29.0)$ & $28(75.7)$ & $<0.001$ \\
\hline
\end{tabular}

$S D$ standard deviation, $B M I$ body mass index, TIA transient ischemic attack, $C T$ computed tomography

$[3,7]$. Therefore, to accurately evaluate the prognosis of patients with poor-grade $\mathrm{SAH}$, predictors other than the initial level of consciousness are needed.
In SAH patients, poor neurological outcome is usually secondary to early brain injury, rebleeding, or delayed cerebral ischemia $[1,8,9]$. Although rebleeding, cerebral 
Table 2 Treatment characteristics

\begin{tabular}{|c|c|c|c|}
\hline & Favorable neurological outcome $(n=186)$ & Poor neurological outcome $(n=37)$ & $p$ value \\
\hline \multicolumn{3}{|l|}{ Aneurysm treatment and timing—no. of patients (\%) } & \multirow[t]{5}{*}{$<0.001$} \\
\hline Early treatment within $72 \mathrm{~h}$ & $174(93.5)$ & $24(64.9)$ & \\
\hline Early but non-aneurysm detection & $8(4.3)$ & $0(0)$ & \\
\hline Late treatment & $1(0.5)$ & $1(2.7)$ & \\
\hline No treatment & $3(1.6)$ & $12(32.4)$ & \\
\hline \multicolumn{4}{|l|}{ Aneurysm management—no. of patients (\%) } \\
\hline Coiling & $108(58.1)$ & $16(43.2)$ & 0.140 \\
\hline Clipping & $66(35.5)$ & $9(24.3)$ & 0.262 \\
\hline Endotracheal intubation during over $24 \mathrm{~h}$ & $38(20.4 \%)$ & $33(89.2 \%)$ & $<0.001$ \\
\hline External ventricular drainage — no. of patients (\%) & $64(34.4)$ & $16(43.2)$ & 0.403 \\
\hline Delayed cerebral ischemia—no. of patients (\%) & $39(21.0)$ & $7(18.9)$ & 0.953 \\
\hline Decompressive craniectomy—no. of patients (\%) & $12(6.5)$ & $8(21.6)$ & 0.008 \\
\hline Barbiturate coma therapy—no. of patients (\%) & $2(1.1)$ & $3(8.1)$ & 0.042 \\
\hline
\end{tabular}

vasospasm, and delayed cerebral ischemia may occur in patients with initially normal ICP, early brain injury within the first $72 \mathrm{~h}$ after the hemorrhage associated with acute consequences of SAH induced sudden intracranial hypertension [1,9]. Severely increased ICP, one of the major mechanisms of early brain injury, leads to decreased cerebral perfusion pressure, cessation of cerebral blood flow, and ultimately global ischemia and edema $[9,10]$. Early brain injury may increase the risk of developing delayed cerebral ischemia $[9,21]$. Therefore, early monitoring of intracranial hypertension may facilitate the prediction of neurological outcomes in patients with SAH. In addition, it may be also used to identify a subgroup of SAH patients with uncontrolled refractory intracranial hypertension, who are at risk of death or delayed neurological impairment and benefit from different management strategies such as decompressive craniectomy or barbiturate coma therapy. Eventually, early monitoring of intracranial hypertension based on the measurement of ONSD may be useful to treat SAH patients.

ONSD has been proposed as an alternative parameter for detection of increased intracranial pressure [11]. The optic nerve is surrounded by cerebrospinal fluid because it is a part of the central nervous system. Therefore, increased ICP is transmitted through the subarachnoid space surrounding the optic nerve within the nerve sheath, especially the retrobulbar segment, unless circulation of cerebrospinal fluid is not blocked [11]. The ONSD measured during the initial brain CT may be correlated with neurologic outcomes after traumatic brain injury [12, 22]. Simultaneous measurement of ONSD with initial $\mathrm{CT}$ and intracranial pressure were correlated, and ONSD was indicative of intracranial hypertension in patients with severe traumatic brain injury $[12,22]$. In this study, ONSD measured with CT was also a good predictive marker associated with ICP and prognosis in SAH patients.

Table 3 The optic nerve sheath diameters and their modifications according to neurological outcomes

\begin{tabular}{|c|c|c|c|}
\hline & Favorable neurological outcome $(n=186)$ & Poor neurological outcome $(n=37)$ & $p$ value \\
\hline $\mathrm{ONSD}_{\text {average }}$ & $5.86 \pm 0.56$ & $6.45 \pm 0.71$ & $<0.001$ \\
\hline $\mathrm{ONSD}_{\max }$ & $5.99 \pm 0.57$ & $6.62 \pm 0.75$ & $<0.001$ \\
\hline ETD average & $22.78 \pm 1.18$ & $22.64 \pm 1.14$ & 0.504 \\
\hline $\mathrm{ONSD}_{\text {index }}$ & $5.15 \pm 0.52$ & $5.70 \pm 0.60$ & $<0.001$ \\
\hline Follow-up ONSD average & $5.77 \pm 0.59$ & $6.47 \pm 0.71$ & $<0.001$ \\
\hline Follow-up ONSD $\max$ & $5.99 \pm 0.57$ & $6.62 \pm 0.75$ & $<0.001$ \\
\hline Follow-up ETD average & $22.89 \pm 1.65$ & $22.65 \pm 1.13$ & 0.295 \\
\hline Follow-up ONSD index & $5.06 \pm 0.55$ & $5.71 \pm 0.53$ & $<0.001$ \\
\hline$\triangle \mathrm{ONSD}_{\text {average }}$ & $0.01 \pm 0.07$ & $-0.01 \pm 0.06$ & 0.139 \\
\hline
\end{tabular}

ONSD optic nerve sheath diameter, ETD eyeball transverse diameter, $\triangle O N S D_{\text {average }}$ follow-up ONSD $_{\text {average }}$ minus initial ONSD $_{\text {average }}$ 


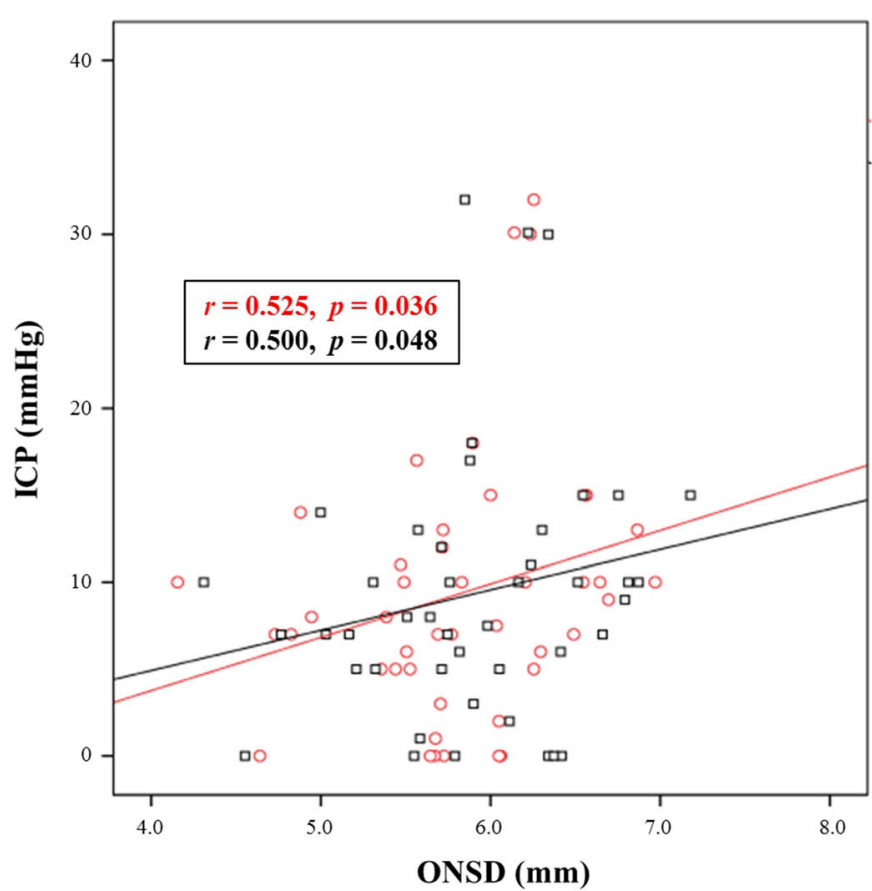

Follow-up ONSD average

$\square$ Follow-up ONSD $_{\text {index }}$

Fig. 3 Scatter plot of intracranial pressure (ICP, $\mathrm{mmHg}$ ) and optic nerve sheath diameter (ONSD, mm). Follow-up ONSDaverage and follow-up $\mathrm{ONSD}_{\text {index }}$ were used in simple correlation analysis

A

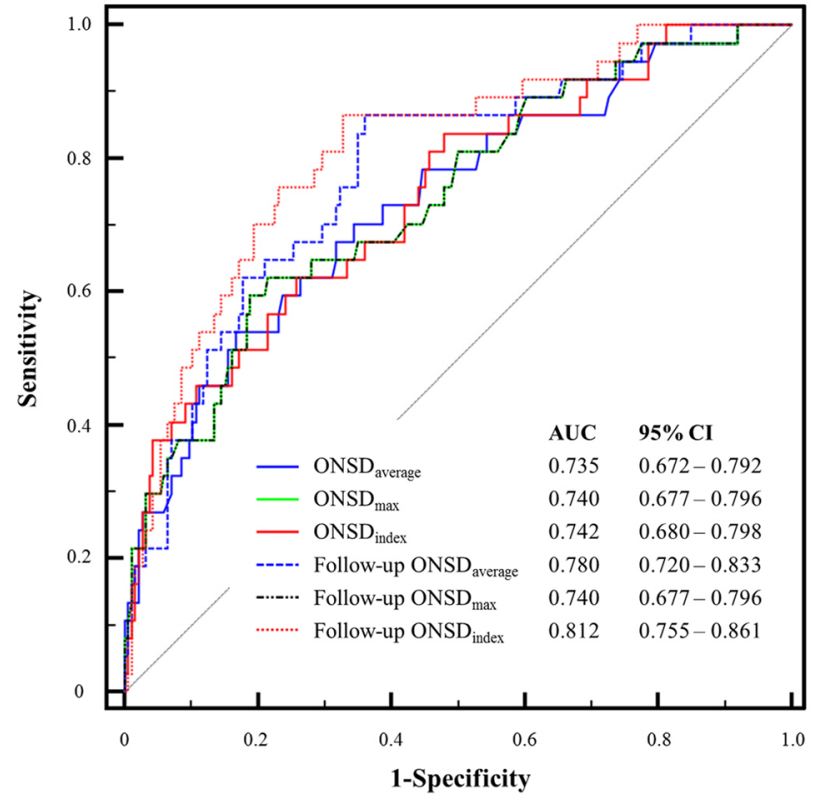

B

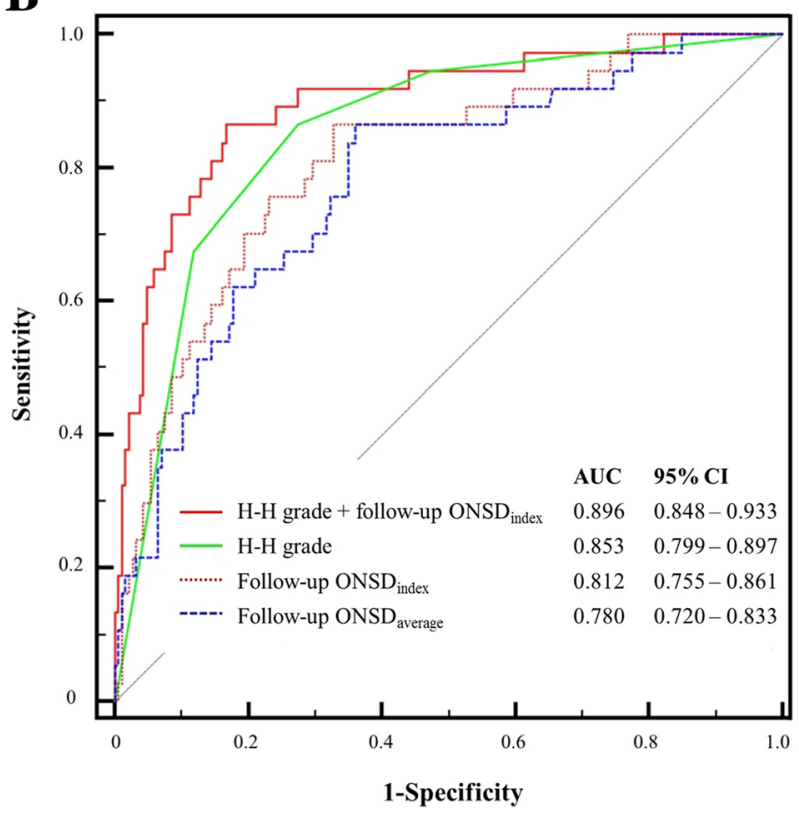

Fig. 4 a, b Receiver operating characteristic curves for predicting poor outcomes using Hunt and Hess grade, the optic nerve sheath diameters, and their modifications (a). Although there were no differences between the areas under the curve (AUCS) of H-H grade, follow-up ONSDaverage, and follow-up ONSD index, the performance of a composite of $\mathrm{H}-\mathrm{H}$ grade and follow-up ONSD index was strongly associated with poor neurological outcomes compared with the use of either marker alone (all $p<0.006)(\mathbf{b})$. Cl confidence interval, ONSD optic nerve sheath diameter, $\mathrm{H}-\mathrm{H}$ grade Hunt and Hess grade 
Although ONSD is considered an indirect marker for ICP, the optimal cutoff for an abnormal ONSD indicating elevated ICP and its associated factors have been unclear [23]. In addition, there are limited reports investigating the role of ONSD modifications or indices in detecting intracranial hypertension compared with absolute ONSD [14, 15]. The ONSD correlates strongly with ETD in healthy people, and the ONSD/ ETD ratio may provide highly reliable data than the absolute ONSD as a marker of ICP [23]. However, in this study, there were no significant differences between the ONSD/ETD ratio and absolute ONSD in predicting poor neurological outcomes. Therefore, future studies with larger cohorts are needed to confirm these findings.

In this study, we evaluated the potential usefulness of ONSD in detecting intracranial hypertension noninvasively and rapidly and determined its value in clinical decision-making for the management of SAH patients. ONSD has been proposed as an indirect and alternative parameter for detection of intracranial hypertension [11, 12]. However, ICP alone is not the only determinant of poor neurological outcome. Therefore, ONSD alone cannot be considered as the ideal predictor of neurological outcomes in SAH patients. Eventually, ONSD may be used in combination with clinical grading scales to improve prognostic accuracy.

This study has several limitations. First, it was a retrospective review of medical records. Therefore, the GOS was also retrospectively determined based on medical records. Second, the non-randomized nature of the registry data may have resulted in selection bias. Although brain CT scans were performed within $48 \mathrm{~h}$ after $\mathrm{SAH}$, a major limitation of this study may be related to the $\mathrm{CT}$ scans performed at different times. Third, the brain CT scan was performed by two different scanners, and ONSDs were also obtained using two different commercial image-viewing software products, which might have resulted in bias. In addition, ONSD measurement requires high image resolution and specific CT settings such as optimal slice thickness and slice angle with the skull base. The heterogeneity of CT scan protocols may explain the relatively weak correlation between ONSD and ICP in this study [24]. Fourth, invasive ICP monitoring was performed in a limited number of patients after aneurysmal treatment. Finally, our study has limited statistical power due to the small sample size. Although it still provides valuable insight, prospective large-scale studies are needed to evaluate the usefulness of ONSD using brain CT in predicting neurological outcomes of patients with SAH to arrive at evidence-based conclusions.

\section{Conclusion}

ONSDs measured with CT may be used to predict neurological outcomes of SAH patients. Furthermore, a composite parameter of $\mathrm{H}-\mathrm{H}$ grade and ONSD may be helpful to more accurately assess the prognosis of SAH patients.

\section{Abbreviations \\ AUC: Area under the curve; CT: Computed tomography; ETD: Eyeball transverse diameter; GOS: Glasgow Outcome Scale; H-H: Hunt and Hess; ICP: Intracranial pressure; IQR: Interquartile range; ONSD: Optic nerve sheath diameter; ROC: Receiver operating characteristic; SAH: Subarachnoid hemorrhage}

\section{Acknowledgements}

We appreciate the excellent statistical support of Keumhee C. Carriere, PhD, and Joonghyun Ahn, MS, of Samsung Biomedical Research Institute.

Funding

Not applicable.

\section{Availability of data and materials}

Regarding data availability, our data are available on the Harvard Dataverse Network (https://doi.org/10.7910/DVN/DVJHBX) as recommended repositories of critical care.

\section{Authors' contributions}

$S L$ participated in the study design, data collection, drafting of the manuscript, and the statistical analysis. YOK participated in the statistical analysis and helped draft the manuscript. JSB participated in the study design and coordination and helped draft the manuscript. JAR participated in the study conception and design, data collection, and drafting of the manuscript. All authors read and approved the final manuscript.

\section{Ethics approval and consent to participate}

This study was approved by the institutional review boards of ChungBuk National University Hospital (CNUH 2018-08-014-001) and Samsung Medical Center (SMC 2018-07-154), and patients' records were reviewed and published according to the Declaration of Helsinki. Informed consent was waived because of the retrospective nature of the study.

\section{Consent for publication}

Not applicable. This study did not contain any individual or personal data in any form (including individual details, images, or videos).

\section{Competing interests}

The authors declare that they have no competing interests.

\section{Publisher's Note}

Springer Nature remains neutral with regard to jurisdictional claims in published maps and institutional affiliations.

\section{Author details \\ ${ }^{1}$ Department of Neurology, ChungBuk National University Hospital, Cheongju, Republic of Korea. ${ }^{2}$ Department of Critical Care Medicine, Samsung Medical Center, Sungkyunkwan University School of Medicine, 81 Irwon-ro, Gangnam-gu, Seoul 06351, Republic of Korea. ${ }^{3}$ Department of Ophthalmology, Konyang University, Kim's Eye Hospital, Myung-Gok Eye Research Institute, Seoul, Republic of Korea. ${ }^{4}$ Department of Neurosurgery, Samsung Medical Center, Sungkyunkwan University School of Medicine, 81 Irwon-ro, Gangnam-gu, Seoul 06351, Republic of Korea.}

Received: 10 December 2018 Accepted: 14 February 2019

Published online: 26 February 2019

\section{References}

1. de Oliveira Manoel AL, Goffi A, Marotta TR, Schweizer TA, Abrahamson S, Macdonald RL. The critical care management of poor-grade subarachnoid haemorrhage. Crit Care. 2016;20:21. 
2. Naval NS, Chang T, Caserta F, Kowalski RG, Carhuapoma JR, Tamargo RJ. Improved aneurysmal subarachnoid hemorrhage outcomes: a comparison of 2 decades at an academic center. J Crit Care. 2013;28:182-8.

3. Konczalla J, Seifert V, Beck J, Guresir E, Vatter H, Raabe A, et al. Outcome after Hunt and Hess grade $V$ subarachnoid hemorrhage: a comparison of pre-coiling era (1980-1995) versus post-ISAT era (2005-2014). J Neurosurg. 2018;128:100-10

4. Wartenberg KE. Critical care of poor-grade subarachnoid hemorrhage. Curr Opin Crit Care. 2011;17:85-93.

5. Hunt WE, Hess RM. Surgical risk as related to time of intervention in the repair of intracranial aneurysms. J Neurosurg. 1968;28:14-20.

6. Teasdale GM, Drake CG, Hunt W, Kassell N, Sano K, Pertuiset B, et al. A universal subarachnoid hemorrhage scale: report of a committee of the World Federation of Neurosurgical Societies. J Neurol Neurosurg Psychiatry. 1988;51:1457.

7. Taylor CJ, Robertson F, Brealey D, O'Shea F, Stephen T, Brew S, et al. Outcome in poor grade subarachnoid hemorrhage patients treated with acute endovascular coiling of aneurysms and aggressive intensive care. Neurocrit Care. 2011;14:341-7.

8. Rowland MJ, Hadjipavlou G, Kelly M, Westbrook J, Pattinson KT. Delayed cerebral ischaemia after subarachnoid haemorrhage: looking beyond vasospasm. Br J Anaesth. 2012;109:315-29.

9. Flynn L, Andrews P. Advances in the understanding of delayed cerebral ischaemia after aneurysmal subarachnoid haemorrhage. F1000Res. 2015;4. https://doi.org/10.12688/f1000research.6635.1. PMID: 26937276.

10. Johnson U, Engquist H, Lewen A, Howells T, Nilsson P, Ronne-Engstrom E, et al. Increased risk of critical CBF levels in SAH patients with actual CPP below calculated optimal CPP. Acta Neurochir. 2017;159:1065-71.

11. Hwan Kim Y, Ho Lee J, Kun Hong C, Won Cho K, Hoon Yeo J, Ju Kang M, et al. Feasibility of optic nerve sheath diameter measured on initial brain computed tomography as an early neurologic outcome predictor after cardiac arrest. Acad Emerg Med. 2014;21:1121-8.

12. Sekhon MS, Griesdale DE, Robba C, McGlashan N, Needham E, Walland K, et al. Optic nerve sheath diameter on computed tomography is correlated with simultaneously measured intracranial pressure in patients with severe traumatic brain injury. Intensive Care Med. 2014;40:1267-74.

13. Legrand A, Jeanjean P, Delanghe F, Peltier J, Lecat B, Dupont H. Estimation of optic nerve sheath diameter on an initial brain computed tomography scan can contribute prognostic information in traumatic brain injury patients. Crit Care. 2013;17:R61.

14. Vaiman M, Gottlieb P, Bekerman I. Quantitative relations between the eyeball, the optic nerve, and the optic canal important for intracranial pressure monitoring. Head Face Med. 2014;10:32.

15. Bekerman I, Sigal T, Kimiagar I, Ben Ely A, Vaiman M. The quantitative evaluation of intracranial pressure by optic nerve sheath diameter/eye diameter CT measurement. Am J Emerg Med. 2016;34:2336-42.

16. DeLong ER, DeLong DM, Clarke-Pearson DL. Comparing the areas under two or more correlated receiver operating characteristic curves: a nonparametric approach. Biometrics. 1988;44:837-45.

17. Schisterman EF, Perkins NJ, Liu A, Bondell H. Optimal cut-point and its corresponding Youden Index to discriminate individuals using pooled blood samples. Epidemiology. 2005;16:73-81.

18. Ruopp MD, Perkins NJ, Whitcomb BW, Schisterman EF. Youden Index and optimal cut-point estimated from observations affected by a lower limit of detection. Biom J. 2008:50:419-30.

19. Chen P, Tebbs JM, Bilder CR. Global goodness-of-fit tests for group testing regression models. Stat Med. 2009;28:2912-28.

20. de Oliveira Manoel AL, Jaja BN, Germans MR, Yan H, Qian W, Kouzmina E, et al. The VASOGRADE: a simple grading scale for prediction of delayed cerebral ischemia after subarachnoid hemorrhage. Stroke. 2015;46:1826-31.

21. Sabri M, Lass E, Macdonald RL. Early brain injury: a common mechanism in subarachnoid hemorrhage and global cerebral ischemia. Stroke Res Treat. 2013;2013:394036.

22. Sekhon MS, McBeth P, Zou J, Qiao L, Kolmodin L, Henderson WR, et al. Association between optic nerve sheath diameter and mortality in patients with severe traumatic brain injury. Neurocrit Care. 2014;21:245-52.

23. Kim DH, Jun JS, Kim R. Ultrasonographic measurement of the optic nerve sheath diameter and its association with eyeball transverse diameter in 585 healthy volunteers. Sci Rep. 2017;7:15906.

24. Monnin P, Sfameni N, Gianoli A, Ding S. Optimal slice thickness for object detection with longitudinal partial volume effects in computed tomography. J Appl Clin Med Phys. 2017;18:251-9.

Ready to submit your research? Choose BMC and benefit from:

- fast, convenient online submission

- thorough peer review by experienced researchers in your field

- rapid publication on acceptance

- support for research data, including large and complex data types

- gold Open Access which fosters wider collaboration and increased citations

- maximum visibility for your research: over $100 \mathrm{M}$ website views per year

At BMC, research is always in progress.

Learn more biomedcentral.com/submissions 